\section{MS40-04 PILATUS3 R CdTe large-area detectors for laboratory applications}

Marcus Müller ${ }^{1}$, Tilman Donath $^{1}$, Michael Rissi $^{1}$, Clemens Schulze-Briese

\section{DECTRIS Ltd.}

email: marcus.mueller@dectris.com

The PILATUS3 X CdTe detector series for synchrotron applications was introduced by DECTRIS in 2015 . Detectors in this series are available with an active area of up to 254 by $298 \mathrm{~mm}^{2}$ and frame rates of up to $500 \mathrm{~Hz}$. This year, DECTRIS introduces the PILATUS3 R CdTe detector series for laboratory applications.

The laboratory series of CdTe detectors features an active area of up to 84 by $106 \mathrm{~mm}^{2}$ and a maximum frame rate of $20 \mathrm{~Hz}$. The large, high-quality CdTe sensor achieves high efficiency not only for $\mathrm{Cu}$, but also Mo and $\mathrm{Ag}$ radiation and the detector is calibrated for use over the entire energy range. Photon counting in each pixel provides noise-free images with no dark signal or readout noise. In contrast to scintillator-based detectors, this direct-conversion CdTe detector exhibits a sharp point-spread function, with counts confined to the 172 by $172 \mu \mathrm{m}^{2}$ pixel of photon incidence.

This presentation will give an overview of the experimental characterization of detector properties such as quantum efficiency, point-spread function, and count rate capability. Furthermore, first results from laboratory diffraction experiments will be presented.

Keywords: Cadmium Telluride, Hybrid Pixel Detectors, Hybrid Photon Counting, Pixel array detectors

\section{MS40-05 Modern CPAD Detector} Technology for Higher Energy X-rays

Holger Ott ${ }^{1}$, Juergen Graf ${ }^{2}$, Tobias Stuerzer ${ }^{1}$, Michael Ruf ${ }^{3}$

1. Bruker AXS GmbH, Karlsruhe, Germany

2. INCOATEC GmbH, Geesthacht, Germany

3. Bruker AXS Inc., Madison, USA

email: holger.ott@bruker.com

Higher energy X-rays result in a compressed diffraction pattern and enable higher resolution to be achieved, which is particularly advantageous for charge density studies or when the diffraction geometry is restricted, for example by a high-pressure cell. Other advantages include strongly reduced extinction and reduced absorption, which is proportional to about $\lambda^{3}$.

Recent advances in X-ray source and mirror technology have provided the home-lab market with viable solutions for higher energy X-rays, namely the $\mathrm{Ag} \mathrm{K} \alpha(0.56089 \AA)$ IuS 3.0 and the In $\mathrm{K} \alpha(0.51359 \AA)$ METALJET. These high flux density systems with small, focused beams overcome the decrease in the absolute scattering power of the crystal, which is proportional to $\lambda^{3}$.

The higher energy X-rays challenge modern detectors, and especially $\mathrm{Si}$-sensor based HPAD detectors. HPAD detectors suffer from low DQE and parallax effects due the thick sensors employed. The latest generation of CPAD (Charge Integrating Pixel Area Detector) on the other hand, with their thin and extremely efficient high Z-element scintillators, overcome these challenges, and provide the ideal solution for shorter wavelength experiments.

This presentation will focus on recent advances in $\mathrm{X}$-ray detector hardware and software development, and will highlight experiments where higher energy X-rays are advantageous, as outlined above.

Keywords: CPAD, detector technology, high pressure, strong absorbers, indium wavelength 